\title{
Fully-Transparent Transmission Surface for Outdoor-Indoor mmWave Coverage Enhancement
}

\author{
Vikrant Singh ${ }^{1}$, Mohsen Khalily ${ }^{1}$, Salman Behboudi Amlashi ${ }^{1}$, J. David Carey ${ }^{2}$, and Rahim Tafazolli ${ }^{1}$ \\ ${ }^{1}$ Institute for Communication Systems (ICS), University of Surrey, Guildford, United Kingdom. \\ Email: vikrant.singh@surrey.ac.uk,m.khalily@surrey.ac.uk, s.behboudiamlashi@surrey.ac.uk, r.tafazolli@surrey.ac.uk \\ ${ }^{2}$ Advanced Technology Institute (ATI), University of Surrey, Guildford, United Kingdom. \\ Email: david.carey@surrey.ac.uk
}

\begin{abstract}
This paper presents a fully-transparent and novel frequency selective surface (FSS) that can be deployed instead of conventional glass to reduce the penetration loss encountered by millimeter wave (mmWave) frequencies in typical outdoorindoor $(\mathrm{O2I})$ communication scenarios. The presented design uses a $0.035 \mathrm{~mm}$ thick layer of indium tin oxide (ITO), which is a transparent conducting oxide (TCO) deposited on the surface of the glass, thereby ensuring the transparency of the structure. The paper also presents a novel unit cell that has been used to design the hexagonal lattice of the FSS structure. The dispersion and transmission characteristics of the proposed design are presented and compared with conventional glass. The presented FSS can be used for both $26 \mathbf{G H z}$ and $28 \mathrm{GHz}$ bands of the mmWave spectrum and offers a lower transmission loss as compared to conventional glass without any considerable impact on the aesthetics of the building infrastructure.

Index Terms-mmWave, transmission surface, frequency selective surface (FSS), transparent conductor (TC), indium tin oxide (ITO).
\end{abstract}

\section{INTRODUCTION}

Wireless communication systems continue to experience exponential growth due to the rapid adoption of digital technology in several sectors. A report from Cisco suggests that by 2023 there will be 29.3 billion networked devices, with nearly two-thirds of the global population having internet access [1]. To ensure that future wireless communication systems are adequately equipped to handle this surge, new and innovative technologies will need to be developed and adopted that are not only smart and efficient but can be deployed easily in commercial networks. The research community will be required to adopt a holistic approach where working on all dimensions, such as improved system capacity, higher spectrum resources, and increased network densification will be necessary.

From the research and initial commercial trials conducted for $5 \mathrm{G}$, it has been observed that the sub- $6 \mathrm{GHz}$ band is insufficient to deliver on all the demands of future communication networks. Therefore a new spectrum pool needs to be adopted, which not only delivers on the capacity front but is also available in sufficient amount to handle the needs of all stakeholders. Millimeter-wave (mmWave) has thus emerged along with massive multiple-input-multiple-output (massive MIMO) and ultra-densification as the three big pillars to support successive future generations of wireless communications [2]. However, mmWaves suffers from high path loss under harsh radio propagation environment such as rain, presence of obstacles and outdoor-indoor (O2I) penetration [2]. Furthermore, due to the rapid variation in channel conditions at mmWave frequencies, it becomes too difficult for the current network architecture to adapt to these variations as presently either the base station (BS) or the user equipment (UE) makes necessary changes to handle these variations. These issues have been a major factor in stalling the rapid adoption of mmWave in stateof-the-art commercial networks on a large scale [2].

For the research community, it has become essential to come up with novel ideas to alleviate the problems faced by the mmWave spectrum in order to accelerate its deployment in commercial networks. One such idea that is rapidly gaining momentum is to equip the radio propagation environment with smart and dynamic entities such as Large Intelligent Surfaces (LISs), also known as Intelligent Reflecting Surfaces (IRSs) or Hypersurfaces (HFSs) [3]-[5]. This will add a new entity to the network architecture which can be used to impart some level of control over the radio propagation environment and can also be used to establish a link between the BS and UE where an adequately strong link or line of sight (LOS) path does not exist. Another dynamic entity that has the potential of assisting mmWave communications is a Frequency Selective Surface (FSS) [6] that can be deployed instead of conventional walls or windows. These surfaces could be designed in such a way that they can interact with or pass the mmWave signals rather than attenuating them. These types of surfaces could play a crucial role in the O2I penetration scenario, as showcased by NTT Docomo in a recent demonstration [7].

This work presents a fully-transparent and novel FSS that can be used to reduce the penetration loss encountered by mmWave frequencies during a typical O2I scenario. Since it is optically transparent, it can be deployed in buildings as windows or glass panes without impacting the aesthetics of the infrastructure. The design with a single layer of transparent conductor has a $1 \mathrm{~dB}$ transmission loss bandwidth of $\approx 1.91 \mathrm{GHz}$ and a $3 \mathrm{~dB}$ transmission loss bandwidth of $\approx 4.6 \mathrm{GHz}$. A design with two layers of transparent conductors, i.e. one layer on each side of the glass, has 
TABLE I: Various parameters of Transparent Conductors and their typical values [8].

\begin{tabular}{|c|c|c|c|c|c|c|c|}
\hline Material & $\begin{array}{c}\text { Sheet } \\
\text { Resistance } \\
(\Omega / \mathrm{sq})\end{array}$ & $\begin{array}{c}\text { Visible } \\
\text { Absorption } \\
\text { Coefficient } \\
(\alpha)\end{array}$ & $\begin{array}{c}\text { Figure of } \\
\text { Merit } \\
\left(\Omega^{-1}\right)\end{array}$ & $\begin{array}{c}\text { Electron } \\
\text { Concentration } \\
\left(\mathrm{cm}^{-3}\right)\end{array}$ & $\begin{array}{c}\text { Work } \\
\text { Function } \\
(\mathrm{eV})\end{array}$ & $\begin{array}{c}\text { Plasma } \\
\text { Wavelength } \\
(\mu \mathrm{m})\end{array}$ & $\begin{array}{c}\text { Resistivity } \\
(\mu \Omega \mathrm{cm})\end{array}$ \\
\hline $\mathrm{Cd}_{2} \mathrm{SnO}_{4}$ & 7.2 & 0.02 & 7.0 & - & 4.2 & $>1.3$ & 130 \\
\hline $\mathrm{ZnO}: \mathrm{F}$ & 5.0 & 0.03 & 7.0 & $2 \times 10^{20}$ & 4.2 & $>2.0$ & 400 \\
\hline $\mathrm{ZnO}: \mathrm{Al}$ (AZO) & 3.8 & 0.05 & 5.0 & - & - & - & - \\
\hline$I n_{2} \mathrm{O}_{3}: \mathrm{Sn}$ (ITO) & 6.0 & 0.04 & 4.0 & $\geq 10^{20}$ & 4.8 & $>1.0$ & 100 \\
\hline $\mathrm{SnO}_{2}: F$ & 8.0 & 0.04 & 3.0 & $4 \times 10^{20}$ & 4.9 & $>1.6$ & 200 \\
\hline
\end{tabular}

a $1 \mathrm{~dB}$ transmission loss bandwidth of $\approx 1.79 \mathrm{GHz}$ and a $3 \mathrm{~dB}$ transmission loss bandwidth of $\approx 4.25 \mathrm{GHz}$. The design is suitable to be used in $\mathrm{n} 257(26.50-29.50 \mathrm{GHz}), \mathrm{n} 258$ (24.25-27.50 GHz), and n261 (27.50-28.35 GHz) bands of the Frequency Range-2 (FR-2) of the 5G-NR, which are rapidly becoming some of the most widely adopted mmWave bands for commercial 5G networks [9].

\section{TRANSPARENT CONDUCTORS}

Transparent conductors (TCs) are a special type of conductors that not only have good electrical conductivity but exhibit high optical transparency [10]. TCs have been used in applications such as touchscreens, flat-panel displays, photovoltaics, aircraft windows, and CubeSat missions [11]. TCs have been prepared using a wide variety of materials, e.g., semiconducting oxides of zinc, tin, cadmium, indium, and metals, e.g., silver, gold, and titanium nitride [8]. An appropriate performance indicator for TCs is the ratio of their electrical conductivity $(\sigma)$ to the visible absorption coefficient $(\alpha)$ [8], this ratio is expressed as shown in (1).

$$
\sigma / \alpha=-\left\{R_{S} \ln (T+R)\right\}^{-1},
$$

where $R_{S}$ is the sheet resistance in ohms per square, $T$ is the total visible transmission, and $R$ is the total visible reflectance. This ratio is known as the "Figure of Merit" and typical values of this ratio are given in Table I for various TCs. Judging the performance of TCs by their figure of merit alone, is not always appropriate as different applications have different requirements in terms of electrical conductivity, optical transparency, physical characteristics, and method of fabrication. Therefore, the choice of TC depends on the requirements of a specific application and not just its performance. A summary of various parameters and their typical values are given in Table I.

\section{A. Electrical Conductivity}

The frequency dependent electrical conductivity $\sigma(\omega)$ depends on the concentration of free electrons and their mobility in a TC. In the case of metals, e.g., silver, gold, and copper, this is governed by the atomic structure and the fixed carrier concentration of the respective metal. However, for semiconductors, the concentration of free electrons is governed by the number of valence electrons in the dopant atoms, and the mobility of these electrons depends on the number of dopant atoms inserted in the semiconductor lattice. Electron concentration for some TCs is summarised in Table I for reference.

\section{B. Plasma Frequency}

High transparency $(>80 \%)$ in the visible region of the spectrum is explained by the appearance of a plasma edge. Light gets reflected due to light frequency $(\omega)$ coinciding with the frequency of collective oscillations of the charge carriers in a material called as plasma frequency $\left(\omega_{p}\right)$, it is given by (2).

$$
\omega_{p}=\left\{\frac{n \cdot e^{2}}{\varepsilon_{0} \cdot \varepsilon_{\infty} \cdot m^{*}}\right\}^{1 / 2},
$$

where $n$ is the concentration of charge carriers, $e$ is the electronic charge, $\varepsilon_{0}$ is the permittivity of free space, $\varepsilon_{\infty}$ is the high-frequency permittivity and $m^{*}$ is the effective electron mass [12]. The plasma frequency for conduction electrons in a TC governs the optical properties [8]. Electrons cannot respond at frequencies higher than plasma frequency, and the material behaves as a transparent dielectric. Whereas, the TC interacts with the impinging electromagnetic waves below plasma frequency. For the majority of TCs, the plasma frequency lies in the near-infrared region of the spectrum. Some typical values of plasma wavelength are given in Table I for reference.

\section{FSS DESIGN AND DISCUSSION}

The unit cell and the complete FSS structure have been designed and simulated using CST Studio Suite $2018^{\circledR}$ to operate at the $26 \mathrm{GHz}$ and $28 \mathrm{GHz}$ mmWave bands. Two versions of the FSS have been designed and compared, one with a conducting surface present on only one side (one-sided FSS) and the other with a conductive surface present on both sides (two-sided FSS). The FSS structure consists of a glass panel with a layer of TC applied on the surface. The TC considered for this design is indium tin oxide (ITO) which is one of the most widely used transparent conducting oxide (TCO) due to its high transparency $(75-92 \%$ at $550 \mathrm{~nm}$ ), good conductivity $(6-12 \Omega / \mathrm{sq})[11]$ and ease with which it can be deposited as a thin film. 


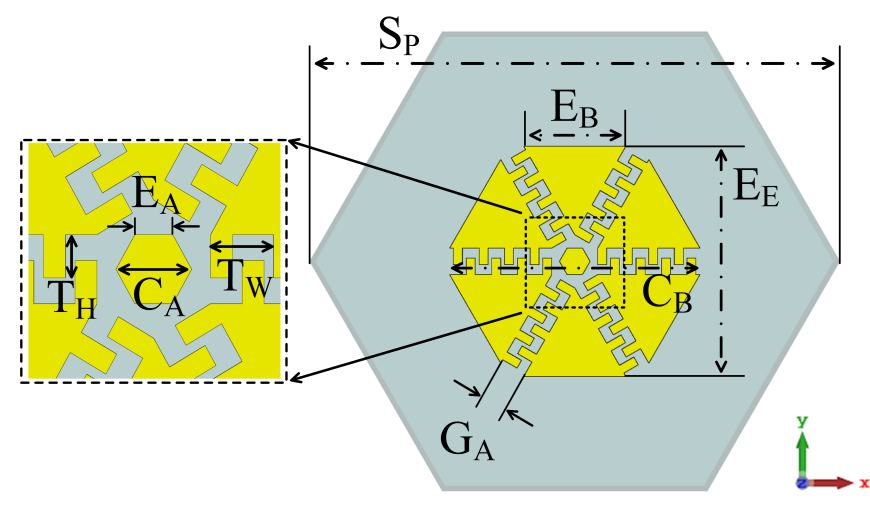

(a)

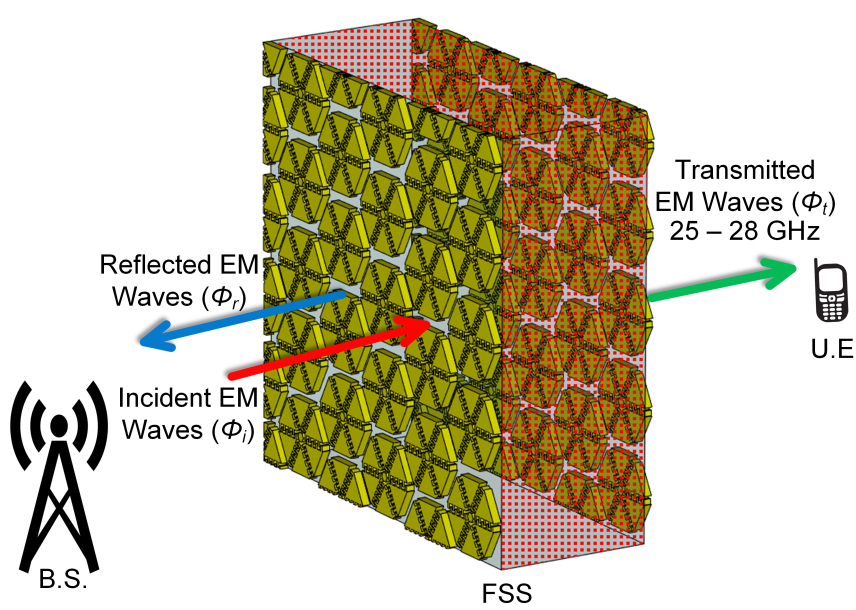

(b)

Fig. 1: Proposed unit cell and FSS. (a) Schematic of the unit cell. (b) Illustration of the proposed FSS being deployed in an O2I scenario.

\section{A. Unit Cell Design}

The proposed unit cell design is shown in Fig. 1a and its design has been inspired from hexagonal geometric shape. The final design parameters of the optimized design are $S_{P}=12 \mathrm{~mm}, E_{B}=1.338 \mathrm{~mm}, E_{E}=3.117 \mathrm{~mm}, C_{B}=$ $4.0 \mathrm{~mm}, G_{A}=0.4 \mathrm{~mm}, C_{A}=0.18 \mathrm{~mm}, E_{A}=0.15 \mathrm{~mm}$, $T_{H}=0.10 \mathrm{~mm}, T_{W}=0.18 \mathrm{~mm}$. The unit cell has been designed using a $10 \mathrm{~mm}$ thick glass substrate with a $0.035 \mathrm{~mm}$ thick layer of ITO applied on its surface. Several unit cells have been arranged in a hexagonal lattice to form the full FSS structure as shown in Fig. 1b. The hexagonal lattice offers better performance as compared to a square lattice in terms of dispersion behaviour [13] and offers high packing density.

\section{B. Simulation Results}

The dispersion diagram of the structure has been calculated and is presented in Fig. 2. The dispersion relation shows that for the proposed unit cell, the relation between frequency and wavenumber does not follow a linear relation, which is usually the case for light or electromagnetic fields in both normal and isotropic medium. The assumptions for the calculation of dispersion relation are based on the eigenvalue solutions of Maxwell's equations. Therefore, an initial hexagonal region is defined on the surface of the unit cell as the full Brillouin zone of the structure. The path for calculating the dispersion relation of the unit cell starts from the center of the Brillouin zone and then traverses towards the edge of the defined Brillouin zone. The complete path for calculation is shown in Fig. 2 and is marked as $\Gamma-\mathrm{M}-\mathrm{K}-\Gamma$, which forms the irreducible Brillouin zone. The dispersion diagram has been calculated for both one-sided FSS scenario and two-sided FSS scenario. The outcome for both the scenarios is the same, this implies that for both the scenarios, the surface propagation mode of the wave can confine the electromagnetic field locally inside the structure. The E-field confinement of the unit cell depicts that maximum confinement can occur at the center of the unit cell as seen in Fig. 2. It can also be observed that the behavior of the dispersion curve is not perfectly asymptotic at just below $26 \mathrm{GHz}$. The reason for this being, even though fundamental propagation modes have weak tangential components (surface propagation modes), but there are some higher-order modes (third propagation modes or higher) with strong tangential components at this frequency causing this behaviour.

The transmission characteristics of the proposed FSS are shown in Fig. 3. They have been calculated for the designed unit cell by applying Floquet's theorem. The transmission curve has been obtained for three scenarios; i) for the FSS structure with a single ITO layer present on one side of the glass, ii) for the FSS structure with an ITO layer present on both sides of the glass, and iii) for glass without any ITO layer present on it. The FSS has a peak transmission at $\approx 27 \mathrm{GHz}$, as observed in Fig. 3. The normalized transmission characteristics

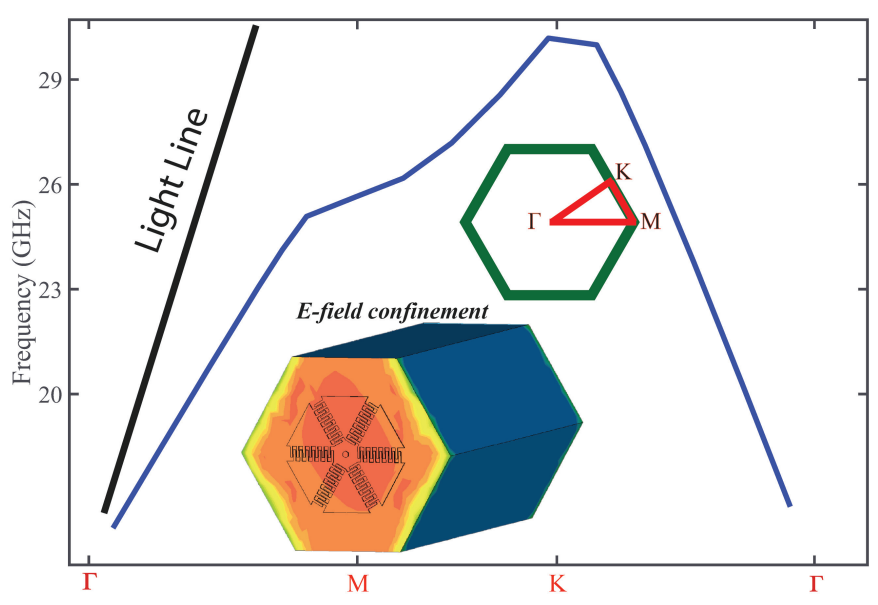

Fig. 2: Dispersion diagram and Brillouin zone of the proposed unit cell. 


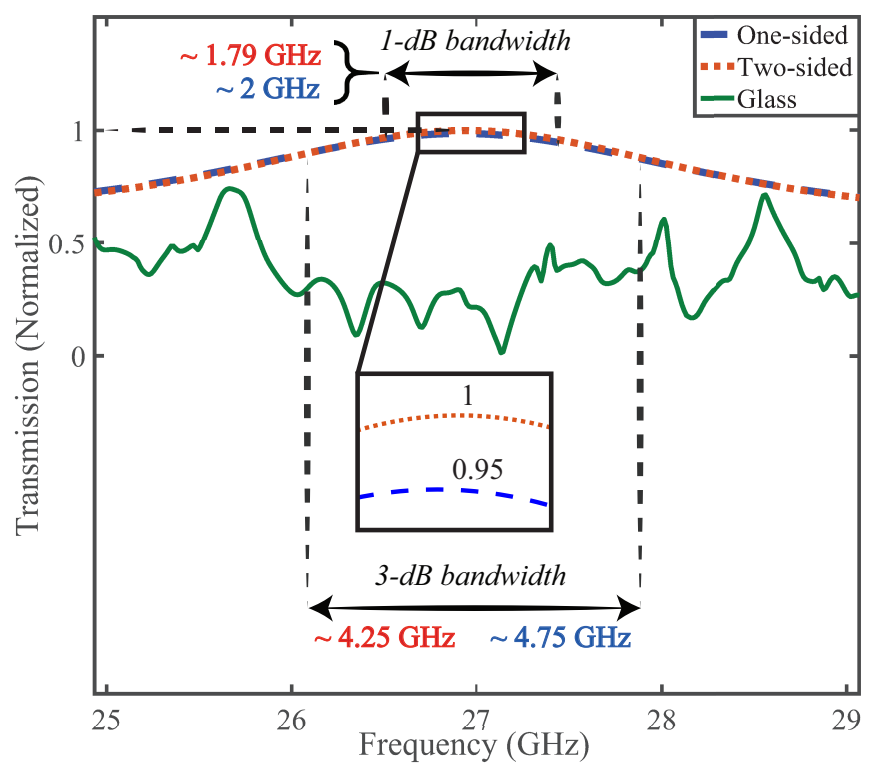

Fig. 3: Transmission characteristics of the proposed structure and comparison with conventional glass.

of the FSS show a higher passage of signal power when compared to normal glass, as shown in Fig. 3. This implies that the proposed FSS structure can alleviate the penetration loss encountered by $25-28 \mathrm{GHz}$ mmWave signals as compared to a conventional glass sheet of similar thickness. Furthermore, two different bandwidths have been defined for the calculated transmission spectra; i) based on $\leq 1 \mathrm{~dB}$ transmission loss and ii) based on $\leq 3 \mathrm{~dB}$ transmission loss. As observed from Fig 3, the two-sided structure allows a better transmission $(\approx 5 \%)$ than a one-sided configuration at the center frequency. However, a trade-off has been observed between transmission loss and bandwidth. In case of a one-sided FSS structure, $1 \mathrm{~dB}$ transmission loss bandwidth is $\approx 1.91 \mathrm{GHz}(26-27.909 \mathrm{GHz})$ whereas for the two-sided structure it is slightly lower and is $\approx 1.79 \mathrm{GHz}(26.027-27.820 \mathrm{GHz})$. Similarly, the $3 \mathrm{~dB}$ transmission loss bandwidth for a one-sided structure is $\approx 4.6 \mathrm{GHz}(24.550-29.148 \mathrm{GHz})$, whereas, for the doublesided structure, it is $\approx 4.25 \mathrm{GHz}(24.716-28.962 \mathrm{GHz})$.

\section{FUTURE WORK}

The results obtained for the proposed design give rise to a range of future opportunities. In addition to using ITO as the TC, the fabrication could also be done using aluminum zinc oxide (AZO). The performance of AZO can be compared with ITO. Furthermore, the designed FSS could be deployed in a mmWave test-bed network, and real-world improvement in mmWave signals and their ultimate impact on the user experience when compared to conventional glass can be measured.

\section{CONCLUSION}

A fully-transparent FSS based on a novel unit cell in a hexagonal lattice arrangement has been designed and compared with conventional glass in terms of penetration loss at mmWave frequencies. The dispersion diagram and the transmission characteristics obtained show that the FSS has good performance for the $26 \mathrm{GHz}$ and $28 \mathrm{GHz}$ mmWave frequency bands. The designed unit cell offers $\approx 1.91 \mathrm{GHz}$ and $\approx 4.6 \mathrm{GHz}$ bandwidth for $\leq 1 \mathrm{~dB}$ and $\leq 3 \mathrm{~dB}$ transmission loss respectively for the one-sided scenario, and it offers $\approx 1.79 \mathrm{GHz}$ and $\approx 4.25 \mathrm{GHz}$ bandwidth for $\leq 1 \mathrm{~dB}$ and $\leq$ $3 \mathrm{~dB}$ transmission loss respectively for the two-sided scenario. The two-sided scenario offers $\mathrm{a} \approx 5 \%$ better transmission performance at the cost of reduced bandwidth when compared with the one-sided scenario. The results also showcase that the proposed FSS has a lower peak transmission-loss when compared to conventional glass for mmWave frequencies. This shows that the proposed design can be considered for carrying out further research to accelerate the deployment of mmWave frequency bands in commercial networks by reducing the penetration loss encountered by mmWave in typical O2I scenarios.

\section{REFERENCES}

[1] "Cisco annual internet report - cisco annual internet report (2018-2023) white paper," Mar 2020. [Online]. Available: https://www.cisco.com/c/en/us/solutions/collateral/executiveperspectives/annual-internet-report/white-paper-c11-741490.html

[2] J. G. Andrews, S. Buzzi, W. Choi, S. V. Hanly, A. Lozano, A. C. K. Soong, and J. C. Zhang, "What will 5g be?" IEEE Journal on Selected Areas in Communications, vol. 32, no. 6, pp. 1065-1082, 2014.

[3] S. Hu, F. Rusek, and O. Edfors, "Beyond massive mimo: The potential of data transmission with large intelligent surfaces," IEEE Transactions on Signal Processing, vol. 66, no. 10, pp. 2746-2758, 2018.

[4] C. Pan, H. Ren, K. Wang, W. Xu, M. Elkashlan, A. Nallanathan, and L. Hanzo, "Multicell mimo communications relying on intelligent reflecting surfaces," IEEE Transactions on Wireless Communications, pp. 1-1, 2020.

[5] C. Liaskos, S. Nie, A. Tsioliaridou, A. Pitsillides, S. Ioannidis, and I. Akyildiz, "Realizing wireless communication through softwaredefined hypersurface environments," in 2018 IEEE 19th International Symposium on "A World of Wireless, Mobile and Multimedia Networks" (WoWMoM), 2018, pp. 14-15.

[6] A. Kesavan, M. Mantash, J. Zaid, and T. A. Denidni, "A dual-plane beam-sweeping millimeter-wave antenna using reconfigurable frequency selective surfaces," IEEE Antennas and Wireless Propagation Letters, vol. 17, no. 10, pp. 1832-1836, 2018.

[7] "Press releases - docomo conducts world's first successful trial of transparent dynamic metasurface." [Online]. Available: https://www.nttdocomo.co.jp/english/info/media_center/pr/2020/ 0117_00.html.

[8] R. G. Gordon, "Criteria for choosing transparent conductors," MRS Bulletin, vol. 25, no. 8, p. 52-57, 2000.

[9] "The wrc series - $26 \mathrm{ghz}$ and $28 \mathrm{ghz}, "$ Oct 2019. [Online]. Available: https://www.gsma.com/spectrum/wp-content/uploads/2019/06/26and-28-GHz-for-5G.pdf

[10] J. Gao, K. Kempa, M. Giersig, E. M. Akinoglu, B. Han, and R. Li, "Physics of transparent conductors," Advances in Physics, vol. 65, no. 6, p. 553-617, 2016.

[11] Z. J. Silva, C. R. Valenta, and G. Durgin, "Optically transparent antennas : A survey of transparent microwave conductor performance and applications," IEEE Antennas and Propagation Magazine, pp. 0-0, 2020.

[12] T. Zinchenko, E. Pecherskaya, and D. Artamonov, "The properties study of transparent conductive oxides (tco) of tin dioxide (ato) doped by antimony obtained by spray pyrolysis," AIMS Materials Science, vol. 6, no. 2, p. 276-287, 2019.

[13] M. Li, S. Xiao, J. Long, and D. F. Sievenpiper, "Surface waveguides supporting both tm mode and te mode with the same phase velocity," IEEE Transactions on Antennas and Propagation, vol. 64, no. 9, pp. 3811-3819, 2016. 